\title{
FLUCONAZOLE NANOGEL: FABRICATION AND IN VITRO EVALUATION FOR TOPICAL APPLICATION
}

\author{
DIVYA ${ }^{1}$, INDERBIR SINGH ${ }^{2}$, UPENDRA NAGAICH ${ }^{*}$
}

1Department of Pharmaceutics, Amity Institute of Pharmacy, Amity University, Noida, Uttar Pradesh 201313, India, ${ }^{2}$ Department of Pharmaceutics, Chitkara University, Jansla, Rajpura, Punjab 140401

"Email: unagaich@hotmail.com

Received: 17 Dec 2020, Revised and Accepted: 29 May 2021

\section{ABSTRACT}

Objective: The aim of this study is to develop and in vitro evaluation of prepared fluconazole nanogel for seborrheic dermatitis

Methods: Fluconazole nanogel was formulated to act against seborrheic dermatitis. The fluconazole nanoparticles were prepared by a simplified evaporation method and evaluated for particle size, entrapment efficiency, and percent in vitro drug release. The nanogel was also characterized based on parameters like particle size, percent entrapment efficiency, shape surface morphology, rheological properties, in vitro release $R^{2}=0.9046$, and release kinetics.

Results: The nanoparticle with a combination of Eudragit RS and Tween 80 showed the best result with particle size in the range of $119.0 \mathrm{~nm}$ to $149.5 \mathrm{~nm}$, with a cumulative percent drug release of $95 \%$ up to $18 \mathrm{~h}$. The formulated nanogel with optimum concentration of HPMC authenticate with particle size $149.50 \pm 0.5$ with maximum drug release $(92.13 \pm 0.32) \%$.

Conclusion: Different percentages of polymers (ethyl-cellulose, eudragit, and tween 80) are used as variable components in the formulation of nanogel. The optimized batch showed good physical properties (flow index, spreadability, and viscosity) along with rapid drug release. Therefore, it can be concluded that nanogel containing fluconazole has potential application in topical delivery.

Keywords: Seborrheic dermatitis, Fluconazole, Nanogel, Topical application

(C) 2021 The Authors. Published by Innovare Academic Sciences Pvt Ltd. This is an open access article under the CC BY license (https://creativecommons.org/licenses/by/4.0/) DOI: https://dx.doi.org/10.22159/ijap.2021v13i4.40531. Journal homepage: https://innovareacademics.in/journals/index.php/ijap

\section{INTRODUCTION}

Seborrheic dermatitis is a topical skin inflammatory disorder mainly characterized by yellow-grey scales and poorly defined erythematous patches. It affects the sebum-rich areas such as skin, scalp, nasolabial folds, ears, eyebrows, and chest [1]. The frequency is more common in men than in women. The occurrence of the lesion also depends on the weather conditions [2,3]. However, sunlight has some evidence to develop seborrheic dermatitis following psoralen+UVA therapy, also known as PUVA. Treatment of seborrheic dermatitis aims at reducing inflammatory processes and decreasing sebum production [4].

Besides, recent studies have revealed that tea tree oil (Melaleuca oil), honey, and cinnamic acid have antifungal activity against Malassezia species, which may be of benefit in the treatment of seborrheic dermatitis [5]. With the wide availability of preparations, including creams, shampoos, and oral formulations, antifungal agents are safe and effective in the treatment of seborrheic dermatitis [6]. This may, in part, be due to an abnormal or inflammatory immune response to these yeasts.

A variety of treatment modalities are available, including the eradication of the fungus, reducing, or treating the inflammatory process, and decreasing sebum production. These included some antifungal agents, corticosteroids, immunomodulators, and keratolytic. The characterization of these classes of drugs such as the anti-inflammatory properties inherent in many of the antifungal agents as well as the keratolytic properties of selenium, zinc, and tar preparations. The azoles including bifonazole, itraconazole, fluconazole, and ketoconazole represent the largest class of antifungals used in the treatment of fungal infection [7].

Antifungal agents are the mainstay of anti-seborrheic therapy, mostly in the azole form. These agents work by inhibiting ergosterol, an important component of the fungal cell wall, via interference with the fungal cytochrome P-450 (CYP 450) system. This causes an increase in the production of sterol precursors, a fungistatic process that does not allow the fungus to grow or reproduce. Fluconazolefirst generation triazole is selected for the treatment of seborrheic dermatitis as it has fewer side effects but the weakest binding to human CYP 450 [4]. The nanogel of the drug Fluconazole shows better results as it has advantages over the simple topical products available in the market [5].

The present investigation aimed at formulating fluconazole nanogel, effective against seborrheic dermatitis. The research was mainly composed of two steps viz. loading of fluconazole to nanoparticle and second to incorporate these nanoparticles to a gel base forming nanogel. Nanogels are three-dimensional hydrogel materials crosslinked with swell polymers, increasing their efficiency to hold water. These are highly hydrophilic, increasing their biocompatibility to load drug molecules.

\section{MATERIALS AND METHODS}

\section{Materials}

Fluconazole was received as a gift sample from Unichem Laboratory, Ghaziabad. Eudragit RS was purchased from Sigma-Aldrich Pvt Ltd. Ethyl-cellulose and tween 80 were purchased from CDH Pvt. Ltd., New Delhi. All the solvents used were of analytical grade.

\section{Methods}

\section{Preparation of fluconazole nanoparticle}

Fluconazole nanoparticles were prepared by using simplified evaporation methods [8]. On the variable of different concentrations of polymers, six formulations were formulated (table 1). The polymers were mixed with a solvent (ethanol), forming an organic phase following the addition of fluconazole. The aqueous phase was prepared separately by dissolving tween 80 with water. Both phases were mixed by adding organic phase dropwise with continuous stirring [9]. The organic phase was later allowed to evaporate overnight. The mixture is then centrifuged to separate the nanoparticles [10]. 
Table 1: Formulation table for preparing fluconazole nanoparticles

\begin{tabular}{lllll}
\hline S. No. & Formulation code & Ethylcellulose (mg) & Eudragit RS 100 (mg) & Tween 80 (\%) \\
\hline 1 & A1 & 450 & - & 0.5 \\
2 & A2 & 450 & - & 1.0 \\
3 & A3 & 450 & -50 & 1.5 \\
4 & A4 & - & 450 & 0.5 \\
5 & A5 & - & 450 & 1.0 \\
6 & A6 & & 1.5 \\
\hline
\end{tabular}

\section{Characterization parameters for fluconazole nanoparticles}

\section{Particle size and polydispersity index (PDI)}

The undiluted sample of nanoparticles were transferred in cuvette and particle size was measured by using dynamic light scattering (DLS) apparatus. The results were analyzed and performed in triplicate [11].

\section{Percentage drug entrapment efficiency (\%)}

$1 \mathrm{ml}$ of nanoparticle sample is taken for centrifugation at $7168 \mathrm{RCF}$ (relative centrifugal force) for $50 \mathrm{~min}$. The supernatant was collected, washed, and filtered through membrane ( 0.45 micron) filter paper. The absorbance of the sample was noted, and the actual entrapped drug was calculated using the below-mentioned formula: The readings were taken in triplicate [12].

$$
=\frac{\begin{array}{c}
\text { Total drug concentration } \\
\text { Totalamountofdrug-Amountofdruginsupernatant }
\end{array}}{\text { Totalamountofdug }} \times 100
$$

\section{In vitro drug release study}

Keshary-Chien cell (K-C) was used for performing in vitro release study. These are thermo-regulated by using a water jacket at $37 \pm 0.5^{\circ} \mathrm{C}$. Phosphate buffer ( $\mathrm{pH}$ 6.5) was used as a receptor medium.
Nanoparticles (equivalent to $1 \mathrm{mg}$ of fluconazole) were placed in the donor compartment. Dialysis membrane 70 (Hi-Media, Mumbai, India) having a pore size of $2.4 \mathrm{~nm}$ and a molecular weight cut-off between 12,000-14,000 Da was used as a donor compartment. At predetermined time intervals, $5 \mathrm{ml}$ of the samples were withdrawn from the receiver compartment and replaced by the same volume of freshly prepared PBS (pH 6.5). The samples were analyzed at the wavelength of $254 \mathrm{~nm}$ using a UV spectrophotometer. The readings were taken in triplicate [13].

\section{Preparation of fluconazole nanogel}

The prepared and optimized nanoparticles were loaded to a hydrogel base to form a fluconazole nanogel. The hydrogel was formulated using ingredients and quantities as mentioned in table 2 . Three polymers were used to optimize the hydrogel formulation, the three polymers used are carbopol 940, HPMC, and methylcellulose. Different concentrations of polymer aqueous solution (antisolvent) were prepared by dispersing the calculated amount of polymer in warm water with constant stirring. Then add the previous mixture containing the drug using a syringe to a polymer mixture followed by constant stirring at 7168 RCF by a homogenizer. Methyl and propylparaben as preservatives were added slowly with continuous stirring until gel formation [14].

\begin{tabular}{|c|c|c|c|c|c|c|c|c|c|}
\hline S. No. & Ingredients & F1 & F2 & F3 & F4 & F5 & F6 & F7 & F8 \\
\hline 1 & Fluconazole (mg) & 1 & 1 & 1 & 1 & 1 & 1 & 1 & 1 \\
\hline 2. & Carbopol 940 & 0.5 & 1 & - & - & - & - & - & - \\
\hline 3. & Methyl cellulose & - & - & 2 & 4 & - & - & - & - \\
\hline 4. & HPMC & - & - & - & - & 1.5 & 2 & - & - \\
\hline 5. & Pectin & - & - & - & - & - & - & 3 & 4 \\
\hline 6. & Glycerine & 10 & 10 & 10 & 10 & 10 & 10 & 10 & 10 \\
\hline 7. & Propylene glycol & 20 & 20 & 20 & 20 & 20 & 20 & 20 & 20 \\
\hline 8. & Methylparaben & 0.03 & 0.03 & 0.03 & 0.03 & 0.03 & 0.03 & 0.03 & 0.03 \\
\hline 9. & Propylparaben & 0.01 & 0.01 & 0.01 & 0.01 & 0.01 & 0.01 & 0.01 & 0.01 \\
\hline 10. & Purified water to & 100 & 100 & 100 & 100 & 100 & 100 & 100 & 100 \\
\hline
\end{tabular}

Table 2: Formulation table for preparing fluconazole nanogel

\section{Characterization of fluconazole nanogel}

\section{Rheological parameters}

\section{Viscosity}

Brookfield viscometer was used to analyze the viscosity of the formulated nanogel. The formulated nanogel was taken in beakers and analyzed by setting spindle number and rpm. The readings were taken in triplicate.

\section{Spreadability}

The gel was evaluated by using a glass slide method. The formulated gel was kept between the two sides within a pre-marked circle (1 $\mathrm{cm})$. The pre-weighted plate was kept above the gel for $5 \mathrm{~min}$ [12] The increase in the diameter due to gel spreading was noted and calculated using the formula mentioned below:

$$
\text { Spreadability }=\text { mass } \times \text { length } / \text { time }
$$

\section{pH determination}

$\mathrm{pH}$ of the nanogel formulation was measured using a $\mathrm{pH}$ meter. For this determination, $1 \mathrm{~g}$ of nanogel was weighed and dispersed in 10 $\mathrm{ml}$ of distilled water. This was kept for 4-5 min for taking the actual $\mathrm{pH}$ value. The reading was taken in triplicate.

\section{Drug content}

Nanogel was dissolved in $10 \mathrm{ml}$ of ethanol and thereby drug content was measured. The mixture was centrifuged at 448 RCF for $1 \mathrm{~h}$. The supernatant liquid was withdrawn, and samples were analyzed using a UV spectrophotometer at $260 \mathrm{~nm}$.

\section{Particle shape and surface morphology}

The particle shape and surface morphology of prepared nanogel was visualized by scanning electron microscopes (Cart Zeiss EV018). One drop of the sample was placed on a slide, and excess water was left to dry at room temperature. The testing slides of samples were prepared by lightly sprinkling samples on double-sided adhesive tape on an aluminum stub. The samples were coated with gold to a thickness of 200 to $500^{\circ} \mathrm{A}$ under an argon atmosphere using a gold sputter module in a high vacuum evaporator. The samples were then randomly scanned, and photomicrographs were taken at different magnifications with SEM [15].

\section{Transmission electron microscopy (TEM)}

TEM was used to visualize (morphology and structure) of nanogel. The samples were dried on a copper grid and adsorbed with filter paper. After drying, the sample was viewed under the microscope at different magnifications at an accelerating voltage of $100 \mathrm{kV}$.

\section{Drug release kinetics studies}

From the characterization parameters one optimized formulation was selected and evaluated for release kinetics to understand the release mechanism of the formulated nanogel. For this, data obtained from in vitro drug release was plotted for zero-order 
equation, first-order equation, Korsmeyer's Peppas equation, and Higuchi's equation.

\section{Drug-excipients compatibility studies}

While formulating fluconazole nanogel, interaction studies were performed. No interaction was found while performing interaction studies using Fourier transform Infrared Spectroscopy technique. The results have been illustrated in fig. 1.

\section{In vitro drug release studies}

The various nanogel batches were evaluated using the KesharyChien cell (K-C) cell. The procedure used for in vitro release study was the same as employed for fluconazole nanoparticles.

\section{RESULTS AND DISCUSSION}

\section{Preparation of fluconazole nanoparticle}

Fluconazole nanoparticles were prepared by the solvent evaporation method in six different batches.

\section{Characterization for fluconazole nanoparticles}

Nanoparticles of fluconazole were successfully prepared and optimized to find out the best formulation which is to be loaded to a hydrogel base. The optimization was based on particle size, percent entrapment efficiency, and in vitro drug release.

\section{Drug-excipients compatibility studies}

Particle size and polydispersity index and percentage drug entrapment efficiency (\%)

The result obtained from particle size analysis and \%EE have been mentioned in table 3 . From the results obtained it can be concluded that Formulation A6 (particle size of $149.50 \pm 0.5 \mathrm{~nm}$ ) shows better \% EE and has optimum percent drug release. The smaller size of nanoparticles will help to increase the permeation of the drug through the biological membrane at the target site indicating its efficiency to deliver the drug through a topical route [16]

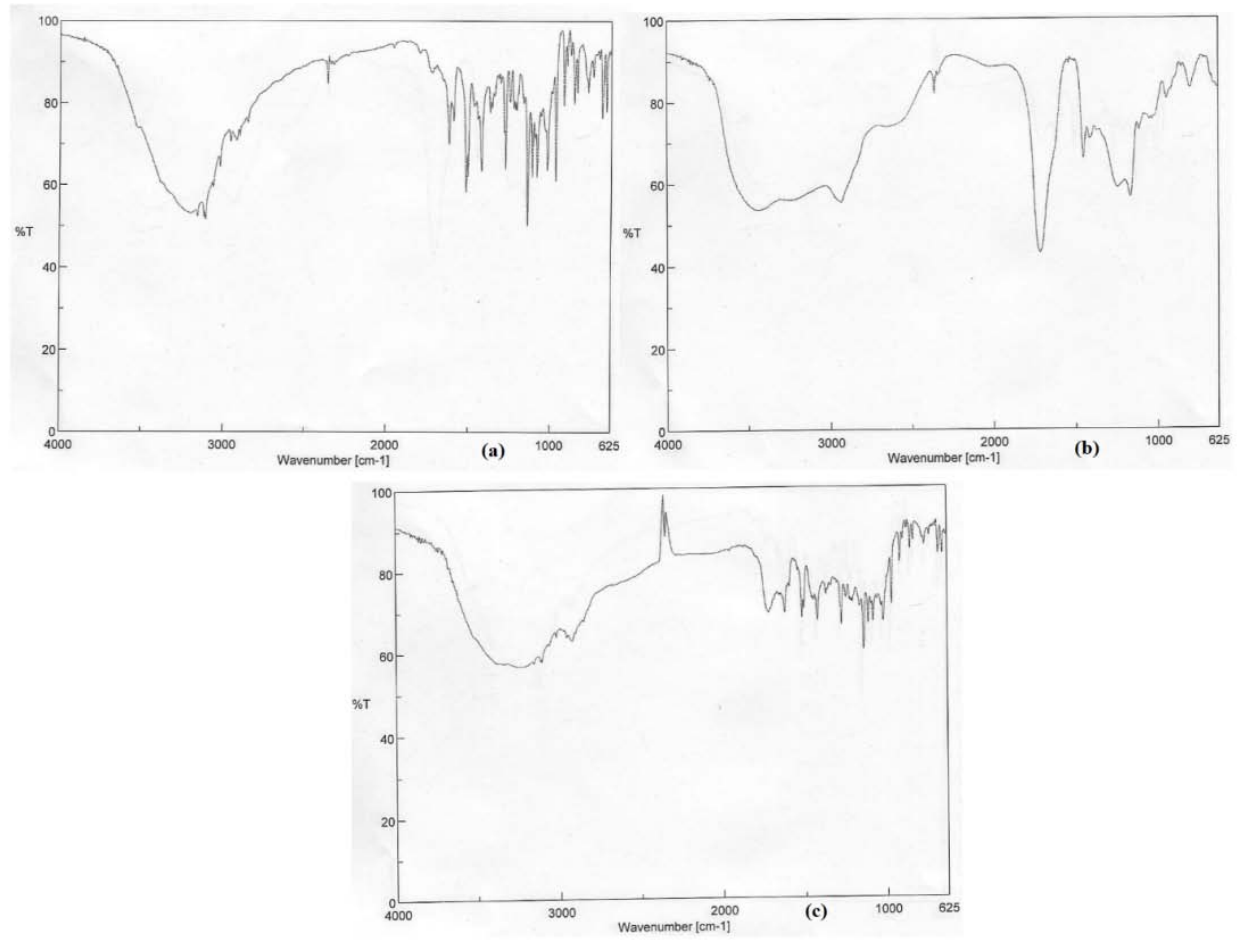

Fig. 1: Fourier transformation infra-red radiation (FTIR) images showing drug-excipient interaction of sample: (a) Drug and excipients, (b) drug (fluconazole), and (c) excipients. No new peak in the sample indicates drug excipient compatibility

Table 3: Particle size and percent entrapment efficiency

\begin{tabular}{lll}
\hline Formulation code & Particle size (nm) & EE\% \\
\hline A1 & $119.46 \pm 0.5$ & $83.66 \pm 0.58$ \\
A2 & $110.83 \pm 0.76$ & $91.36 \pm 1.1$ \\
A3 & $140.83 \pm 1.04$ & $79.50 \pm 0.88$ \\
A4 & $150.36 \pm 0.47$ & $94.20 \pm 0.88$ \\
A5 & $135.93 \pm 0.95$ & $95.80 \pm 0.55$ \\
A6 & $149.50 \pm 0.5$ & $96.03 \pm 0.96$ \\
\hline
\end{tabular}

\section{In vitro drug release of fluconazole nanoparticles}

The in vitro drug release obtained from fluconazole nanoparticles has been depicted in fig. 2. The optimized formulation (A6) was selected and further loaded to a nanogel base.

\section{Characterization of fluconazole nanogel}

An optimized formulation of fluconazole nanoparticles was successfully loaded to form nanogel.
Results obtained from physical and rheological parameters have been depicted in table 4 , which concluded that the formulation exhibited good viscosity, spreadability, and an optimum $\mathrm{pH}$. The formulation was having a good viscosity, spreadability, homogeneity, $\mathrm{pH}$, and visual inspection. The result obtained showed that the formulation is having an optimum viscosity and spreadability. Rheological properties such as flow index, flow behavior, and Coefficient of determination have been illustrated in table 4. 


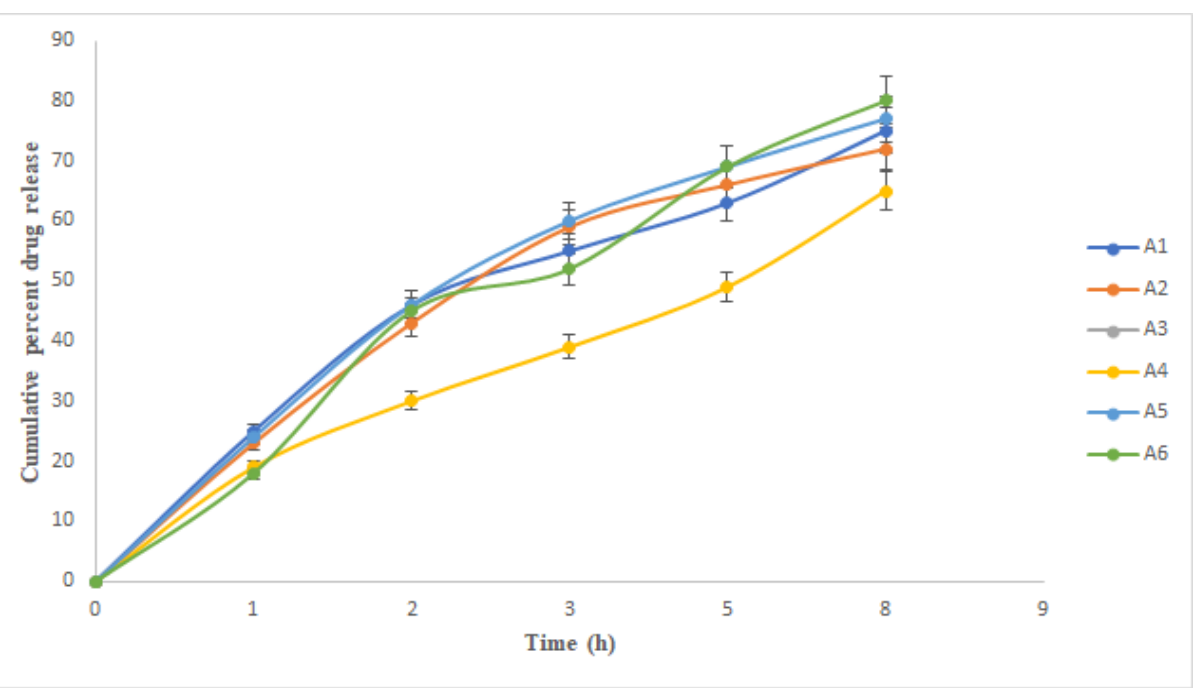

Fig. 2: Cumulative percent in vitro drug release of fluconazole nanoparticles showing that half of the nanoparticles are released in the first $2 \mathrm{hr}$ and $90 \%$ release occurs in $8 \mathrm{hr}$. Among all batches, the $\mathrm{A6}$ batch showed maximum cumulative release with $149.50 \pm 0.5$ particle size and $96.03 \pm 0.96$ entrapment efficiency and $n=3$, mean $\pm S D$

Table 4: Evaluation of physical and rheological properties of fluconazole nanogel batches

\begin{tabular}{llllllll}
\hline Batches & \multicolumn{2}{l}{ Physical properties } & \multicolumn{3}{l}{ Rheological properties } \\
\cline { 2 - 7 } & Colour & $\begin{array}{l}\text { Spreadability } \\
\text { (cm) }\end{array}$ & $\mathbf{p H}$ & $\begin{array}{l}\text { Coefficient of } \\
\text { determination }\end{array}$ & $\begin{array}{l}\text { Flow } \\
\text { index }\end{array}$ & $\begin{array}{l}\text { Viscosity* } \\
\text { ( } \boldsymbol{\eta} \text { ) }\end{array}$ & Flow behavior \\
\hline F1 & Shiny transparent & $4.5 \pm 0.21$ & $6.1 \pm 0.2$ & $0.916 \pm 0.04$ & $0.238 \pm 0.00$ & $1709 \pm 92.1$ & Shear-thinning \\
F2 & Shiny transparent & $4 \pm 0.95$ & $5.99 \pm 0.29$ & $0.9291 \pm 0.04$ & $0.235 \pm 0.01$ & $1918 \pm 47.9$ & Shear-thinning \\
F3 & Transparent & $4.5 \pm 0.2$ & $6.1 \pm 0.12$ & $0.9976 \pm 0.04$ & $0.225 \pm 0.01$ & $1449 \pm 71.8$ & Shear-thinning \\
F4 & Transparent & $3.6 \pm 0.15$ & $6.13 \pm 0.8$ & $0.9823 \pm 0.04$ & $0.210 \pm 0.01$ & $2083 \pm 98.1$ & Shear-thinning \\
F5 & Pale, translucent & $5 \pm 0.23$ & $5.60 \pm 0.15$ & $0.908 \pm 0.04$ & $0.230 \pm 0.00$ & $1012 \pm 48.2$ & Shear-thinning \\
F6 & Pale, translucent & $5 \pm 0.2$ & $5.67 \pm 0.14$ & $0.908 \pm 0.04$ & $0.230 \pm 0.01$ & $1036 \pm 50.6$ & Shear-thinning \\
F7 & Opaque, buff & $5 \pm 0.22$ & $3.6 \pm 0.9$ & $0.9304 \pm 0.04$ & $0.121 \pm 0.00$ & $1247 \pm 61.4$ & Shear-thinning \\
F8 & Opaque, buff & $3.5 \pm 0.12$ & $3.7 \pm 1.2$ & $0.9819 \pm 0.04$ & $0.143 \pm 0.00$ & $2289 \pm 110.3$ & Shear-thinning \\
\hline
\end{tabular}

\section{Drug content}

The formulated fluconazole nanogel was evaluated for drug content parameters. The drug content was found to be in the range of $75.30 \pm 0.43 \%$ to $95.33 \pm 0.49 \%$. The results have been shown in table 5 .

Table 5: Drug content of fluconazole nanogel batches

\begin{tabular}{ll}
\hline Formulations & Drug content (\%) \\
\hline F1 & $85.43 \pm 0.45$ \\
F2 & $77.33 \pm 0.49$ \\
F3 & $80.23 \pm 0.58$ \\
F4 & $94.13 \pm 0.32$ \\
F5 & $92.13 \pm 0.32$ \\
F6 & $93.46 \pm 0.45$ \\
F7 & $95.33 \pm 0.49$ \\
F8 & $75.30 \pm 0.43$ \\
\hline
\end{tabular}

\section{SEM}

The nanogel was visualized by scanning electron microscopy (SEM). They were found to be spherical, having a smooth surface (fig. 3). The average range of particle size was found to be $101.9 \mathrm{~nm}$ to 127.5 $\mathrm{nm}$, having the particle size in the optimal range, which is having excellent morphological properties with minimum toxicity [17].

\section{TEM}

The sample was viewed under the microscope at different magnifications at an accelerating voltage of $100 \mathrm{kV}$. The prepared nanoparticles, showing the spherical shape and particle size of 100$130 \mathrm{~nm}$.

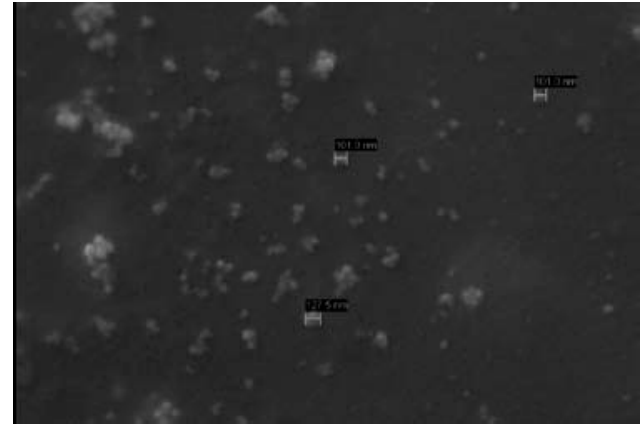

Fig. 3: SEM(scanning electron microscopy) of optimized fluconazole nanogel batch showing the range $\pm 113.6 \mathrm{~nm}, \mathrm{n}=3$, mean \pm SEM
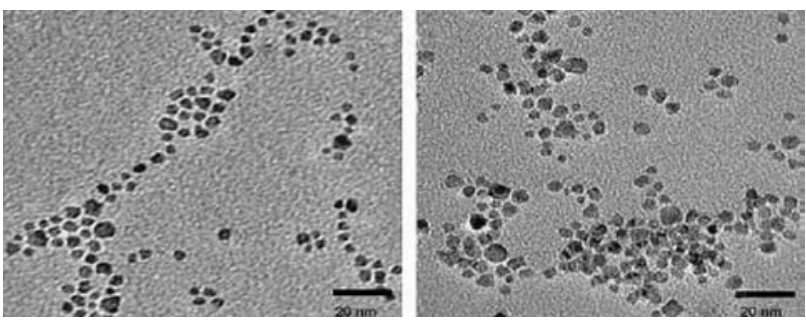

Fig. 4: TEM (transition electron microscopy) of fluconazole optimized nanogel formulation showing the spherical shape and size of average $\pm 112 \mathrm{~nm}, \mathrm{n}=3$, mean \pm TEM 


\section{In vitro drug release}

The in vitro release study showed the release of above $90 \%$ up to 18 h. (fig. 5). The model or equation that best fits the release data was evaluated by correlation coefficient $(\mathrm{r})$ and the value of $\mathrm{n}_{\mathrm{s}}$ particularly for Korsmeyer Peppas's Equation. The release study was best explained by Higuchi's equation, shown in fig. 6 . From the above, it is indicated that the drug release will be diffusion controlled, depending on the swelling criteria of cross-linked polymers present in the hydrogel

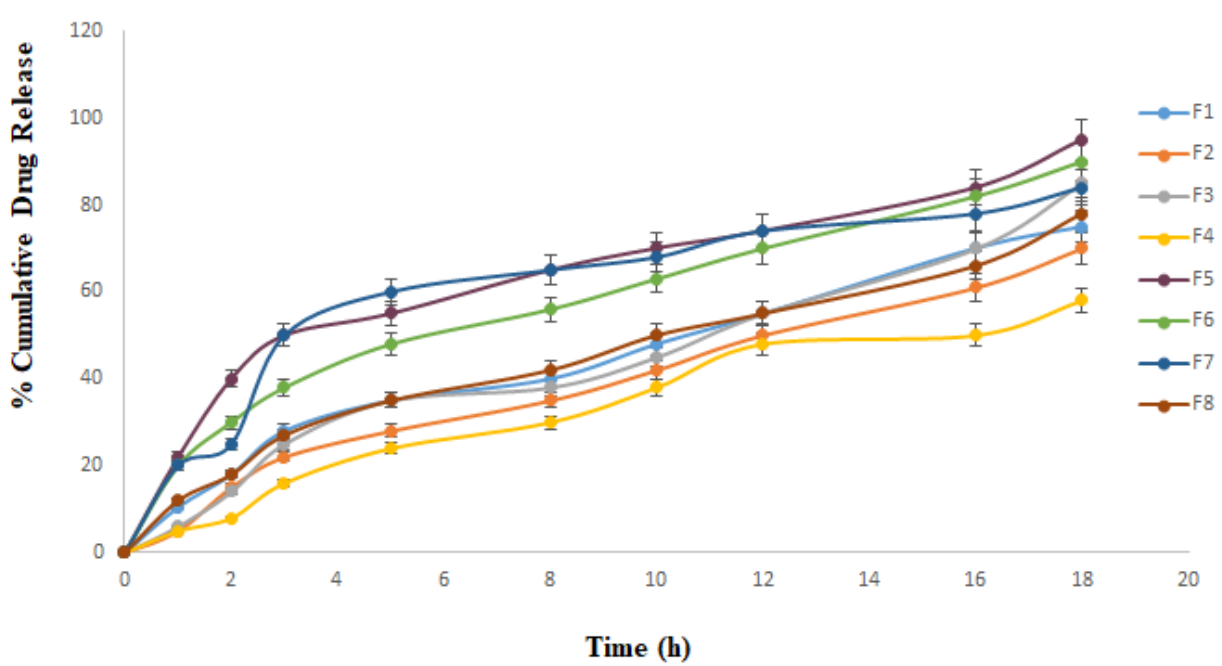

Fig. 5: Cumulative percent in vitro drug release of F1-F8 batches of fluconazole nanogel shows around $50 \%$ of drugs were released in the starting first $10 \mathrm{~h}$ of total release and $90 \%$ drug releases in $18 \mathrm{~h}$, with maximum release of $\mathrm{F} 5, \mathrm{n}=3$, mean $\pm \mathrm{SD}$
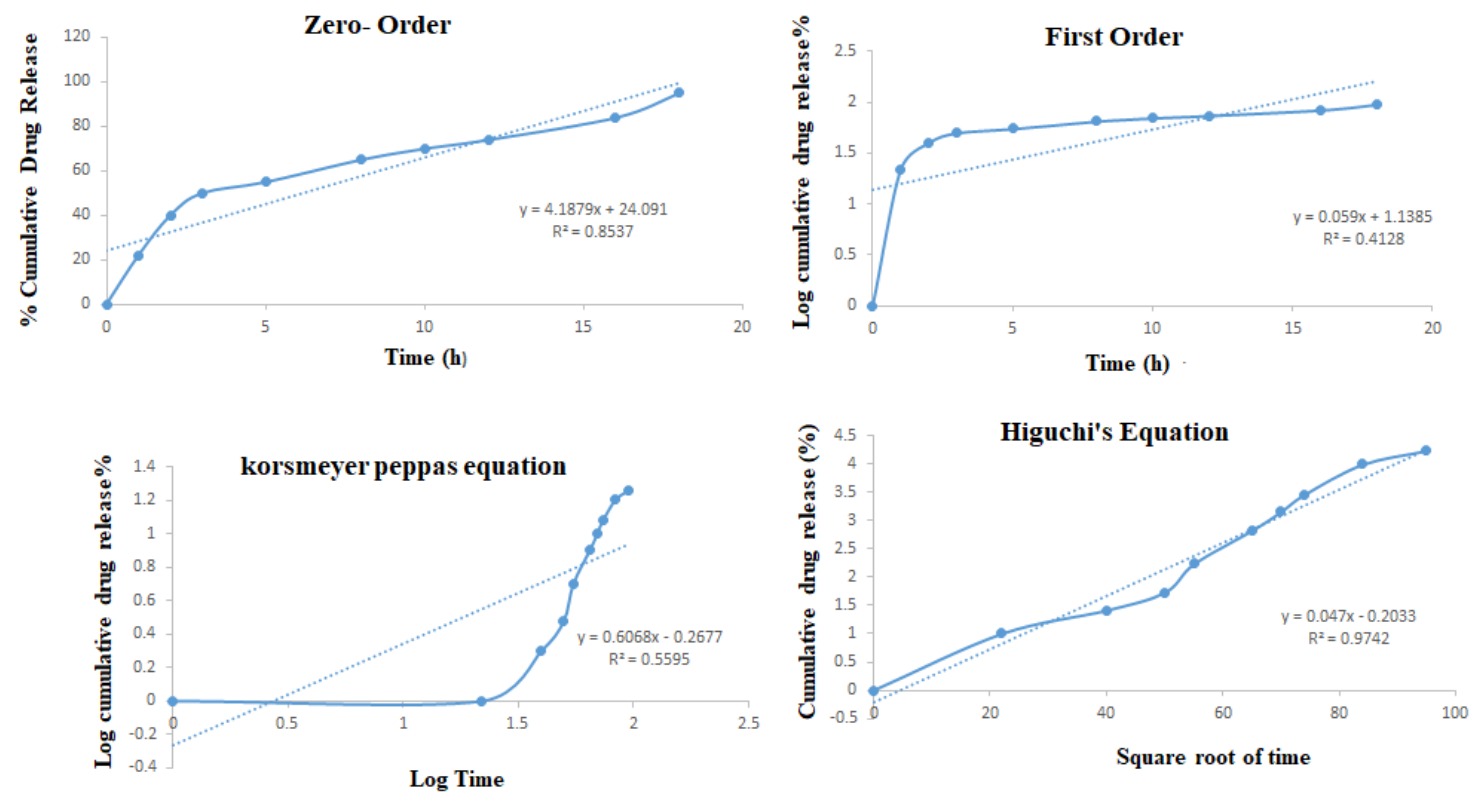

Fig. 6: Drug release kinetics of optimized fluconazole nanogel showed the highest linearity was $R^{2}=0.971$, followed by zero-order $\left(R^{2}=\right.$ $0.853)$, Korsmeyer-Peppas model $\left(R^{2}=0.559\right)$, and first-order equation $\left(R^{2}=0.412\right)$. All values are expressed as, $n=6$, mean $\pm S D$

\section{CONCLUSION}

Fluconazole was successfully incorporated into a gel base. The nanogel shows a good flow index, spreadability, and viscosity. The release profile shows drug release for a prolonged period. The nanogel attains a good percentage of drug content in the formulation. Therefore, it can be concluded that nanogel containing fluconazole has potential application in topical delivery.

\section{ACKNOWLEDGMENT}

The authors wish to thank Founder President Dr. Ashok K. Chauhan, Amity University for their kind support during research.

\section{AUTHORS CONTRIBUTIONS}

All the authors have contributed equally.

\section{CONFLICT OF INTERESTS}

Declared none

\section{REFERENCES}

1. Manriquez JJ, Uribe P. Seborrhoeic dermatitis. BMJ Clin Evid 2007;1:1713.

2. El-Housiny S, Shams Eldeen MA, El-Attar YA. Fluconazoleloaded solid lipid nanoparticles topical gel for treatment of 
pityriasis versicolor: formulation and clinical study. Drug Delivery 2018;1:78-90.

3. Del Rosso JQ. Adult seborrheic dermatitis: a status report on practical topical management. J Clin Aesthet Dermatol 2011;5:32-8.

4. Nickie D, Greer. Voriconazole: the newest triazole antifungal agent. Baylor University Medical Center Proceedings 2003:2:241-8.

5. Berk T, Scheinfeld N. Seborrheic dermatitis. P T 2010;6:348-52.

6. Borda LJ, Wikramanayake TC. Seborrheic dermatitis and dandruff: a comprehensive review. J Clin Investig Dermatol 2015;2:10.

7. Alipour S, Shirooee A, Ahmadi F. Porogen effects on aerosolization properties of fluconazole loaded plga large porous particles. Int J Appl Pharm 2020;4:258-63.

8. Mubarak Al, Hibah. Fluconazole nano-particles loaded gel for improved efficacy in treatment of oral candidiasis. Int J Pharmacol 2019;3:436-40.

9. Abdellatif, Ahmed AH. Hydrogel containing PEG-coated fluconazole nanoparticles with enhanced solubility and antifungal activity. J Pharm Innov 2018;2:112-22.

10. Garala K, Joshi P, Shah M, Ramkishan A, Patel J. Formulation and evaluation of periodontal in situ gel. Int J Pharm Investig 2013;1:29-41.
11. Nagaich U, Gulati N, Chauhan S. Antioxidant and antibacterial potential of silver nanoparticles: biogenic synthesis utilizing apple extract. J Pharm 2016;8:209-18.

12. Goyal R, Macri LK, Kaplan HM, Kohn J. Nanoparticles and nanofibers for topical drug delivery. J Controlled Release 2016;4:77-92.

13. Bashir S, Teo YY, Naeem S, Ramesh S, Ramesh K. pH responsive $\mathrm{N}$-succinyl chitosan/Poly (acrylamide-co-acrylic acid) hydrogels and in vitro release of 5-fluorouracil. PLoS One 2017;7:e0179250.

14. Chauhan S, Gulati N, Nagaich U. Fabrication and evaluation of ultra deformable vesicles for atopic dermatitis as topical delivery. Int J Polym Mater 2019;5:266-77.

15. Parhi R. Cross-linked hydrogel for pharmaceutical applications: a review. Adv Pharm Bull 2017;4:515-30.

16. Kumar A, Pandit V, Nagaich U. Therapeutic evaluation of chemically synthesized copper nanoparticles to promote fullthickness excisional wound healing. Int J Appl Pharm 2020;6:136-42.

17. Savage DT, Hilt JZ, Dziubla TD. In vitro methods for assessing nanoparticle toxicity. Methods Mol Biol 2019;1894:1-29. 